\title{
Achieving Efficiency in Gas Pipeline Connection: Evidence from Ghana
}

\author{
Anthony Kudjo Gborgenu*, Frank B. K. Twenefour ${ }^{1 \dagger}$, Mathias Gyamfi ${ }^{*}$
}

\begin{abstract}
The demand for the use of natural gas is on the increase as an energy source. Natural gas transportation requires a continuous pipeline network from the source of gas across long distance to the various destinations. The main objective involves extending gas pipelines from Takoradi to all the regional capital towns in Ghana to meet the growing demands of its citizenry in order to provide economy and efficiency with regards to cost and environmental sustainability by developing a straight forward method of locating pipeline facilities and designing pipeline networks. The problem is formulated as a network of distances and the solution is presented based on Prim's Algorithm for minimum connections. Data on distances are obtained from the Ghana Highways Authority. The total distance covered by the pipe line network if the existing road networks were used from Takoradi to all the regional capitals towns in Ghana is 5,094km. After Prim's Algorithm was used, the total distance covered decreased to $1,590 \mathrm{~km}$ which is about $68.8 \%$ reduction in the distance covered with regards to cost and the environmental damage caused by construction of pipelines (soil, forest, rivers, wetlands, noise from compressor stations during pipeline discharge and risk of pipeline leakage).
\end{abstract}

Keywords: Natural gas, network analysis, pipelines, Prims algorithms, transportation.

JEL Codes: C6, C61, L95, Q21, N5.

Available Online: 20-06-2016.

This is an open access article under Creative Commons Attribution 4.0 License, 2016.

\section{$1.0 \quad$ INTRODUCTION}

The West Africa Gas Pipelines Project from Nigeria down to Ghana-Takoradi was developed to facilitate the free flow of the Gas. The recent oil find in the western region of Ghana has contributed to the production of the Natural Gas. This gas has to be made available at a cheaper cost to all consumers. This can only be done when natural gas is transmitted from the source to the consumers via pipelines at a minimum cost. The pipelines are connected in network form to enable the free flow of the natural gas from one place to another (WAGPCo, 2004a). To minimize these network connections, constitute the domain of shortest path problems for a trans-regional gas pipeline that provides economy and efficacy.

\footnotetext{
* Department of Mathematics and Statistics, Takoradi Polytechnic, Takoradi, Ghana.

† Corresponding Author's E-mail: frank.twenefour@tpoly.edu.gh
} 
The shortest path problems are the most fundamental and commonly encountered problems in the study of transportation and communication network (Hwee, 2007).

The proposed connection of Gas Pipelines from Takoradi to the other nine regional capitals is being designed to make natural gas from the West African Gas Pipeline (WAGPCo, 2004b) and the Jubilee Oil Field abundantly (JOF) and cheaply to the Ghanaian consumers. Ghanaians are increasing depending on natural gas for its energy needs. In 2010 alone, the nation consumed 124.1 billion cubic metres with demand of 165.2 billion cubic metres of natural gas, forecast for 2015, Business Monitor International (BMI). Large portions of the gas consumed are transported from Takoradi to the rest of the regional capital towns (WAGPCo, 2010).

Ghana is yet to have a major natural gas production basins and the pipeline network of the gas is not fully developed to other regional capitals. Most of the natural gas consumed are imported and few supply from the West African Gas pipeline (WAGP). There is no transmission of pipeline system connecting the West African Gas Pipeline from Takoradi to other nine regional capitals. The primary function of the transmission of gas pipeline is to move huge amounts of natural gas, hundreds of miles from the producing region to the local natural gas utility delivery points (WAGPCo, 2004a). This delivery points, called "city gates stations" are usually owned by distributions companies, although some are owned by transmission companies. Compressor stations at required distances boost the pressure that is lost through friction as the gas moves through the steel pipes (Donkoh et al., 2011).

The transmission segment of the gas industry is responsible for transporting natural gas from the producer to the market areas via pipelines. The transmission segment is composed of pipelines, compressor stations, city gate stations, and storage facilities (Wikipedia, 2008). The energy sector of Ghana is faced with urban and rural areas, transport sector, and industries total dependence of natural gas. This gas is either not readily available or transportation is very high hence high cost of the gas. The connection of gas pipelines from Takoradi to the other regional capitals seek to solve the transportation problem. The installation of these pipelines across these regional capitals is expensive and disruptive to the natural environment. Thus, this thesis seeks to find the shortest-path these pipelines should be installed so as to reduce the cost of installation, minimize the number of pipelines needed and hence a reduction on the environmental problems. The demand for natural gas in Ghana has increased significantly and has been a cheaper alternative fuel used in homes, industries, mining and transportation sectors within the country.

This research seeks to develop a straightforward method of locating pipeline facilities; extend gas pipelines from Takoradi to the regional capital towns in Ghana; design pipeline networks to minimize cost and distance covered and develop a method of incorporating environmental considerations in pipeline network design with a possible solution for a trans-regional gas pipeline for efficiency and economy with regards to cost and environmental damage associated to the construction of pipelines (soil, forest, rivers, wetlands, noise from compressor stations during pipeline discharge and risk of pipeline leakage).

\subsection{MATERIALS AND METHODS}

Shortest path problems are the most fundamental and most commonly encountered problem in the study of transportation and communication networks. The shortest path problem arises when trying to determine the shortest, cheapest or the most reliable path between one or many pairs of nodes in a network. More importantly, algorithms for a wide variety of combinatorial optimization problems such as vehicle routing and network design often called the solution of large number of shortest path problems as subroutines (Arogundade and Akinwale, 2009).

\subsection{MODELING THE PROBLEM}

We used the Prim minimal spanning tree algorithm for directed graph $G=(V, E]$, where $V$ is the set of nodes and $E$ is the set of edges. Each edge is defined by a node pair $(h, k)$, each edge has a non-negative 
length. We start at a given node and sequentially add an edge to tree at each of the iterations. The weight of the graph is the total distance covered in the regional capitals (Prim, 1957).

Therefore, the weight of the graph is given by;

$$
\omega(Q)=\sum_{(h, k) \in Q} \omega(h, k)
$$

More formally, we define a graph $\mathrm{G}$ as an ordered pair $\mathrm{G}=(\mathrm{V}, \mathrm{E})$ where

- $V$ is a set of vertices (nodes)

- $\quad E$ is a set of edges (links).

- Each edge is a pair of vertices. In other words, each element of $E$ is a pair of elements of $V$.

A connected weighted graph with vertices $V(A$ to $X)$ and edges $E$.

Let the vertices V of map $=\{A, B, C, D, E, F, G, H, I, J, K, L, M, N, O, P, Q, R, S, T, U, V, W, X, Y, Z$.

Edges (E) of map= pair (h, k); where $h=A, B, C, \ldots, Z$; and $k=A, B, C, \ldots, Z$.

The algorithm continuously increases the size of a tree, one edge at a time, starting with a tree consisting of a single vertex, until it spans all vertices (Prim, 1957).

- Input: A non- empty connected weighted graph with vertices $\mathrm{V}$ and edges $\mathrm{E}$ in which the weights are non-negative.

- Initialize: $V_{\text {new }}=\{A\}$, where $A$ is an arbitrary node (starting point) from $V, E_{n e w}=\{\}$

- Repeat until $V_{\text {new }}=Z$ :

$\circ$ Choose an edge $(h, k)$ with minimal weight such that $h$ is in $V_{\text {new }}$ and $k$ is not (if there are multiple edges with the same weight, any of them may be picked)

$\circ \quad$ Add $k$ to $V_{\text {new }}$, and $(h, k)$ to $E_{\text {new }}$.

- Output: $V_{\text {new }}$ and $E_{\text {new }}$ describe a minimal spanning tree

Weight function $\boldsymbol{\omega}: \mathbf{E} \rightarrow \mathbf{R}$ (assigning length to edges)

Minimum spanning tree: tree that connects all the vertices and minimizes

$$
\omega(Q)=\sum_{(h, k) \in Q} \omega(h, k)
$$

Since the connection problem is a network problem, it can be solved by the shortest path method. But because of its special nature, it can be solved more easily by Prim's algorithm, which is more efficient for the connection problem.

\subsection{PRIM'S ALGORITHM}

Prim's algorithm solves the shortest path problem for a directed graph with non-negative edge weights. For example, if the vertices of the graph represent gas well or roads connecting the towns and edge weights represent distances between pair of wells (or towns) connected by a pipeline, this algorithm can be used to find the shortest route between two gas wells (or towns). The algorithm builds a spanning tree from scratch by fanning out from a single node and adding arc one at a time. It means a tree spanning on a subset $\mathrm{S}$ of nodes and a nearest neighbour to $\mathrm{S}$. the algorithm does so by identifying an $\operatorname{arc}(\mathrm{i}, \mathrm{j})$ of minimum cost in the cut $[S, S]$. It adds arc $(i, j)$ to the tree, node $j$ to $S$ repeats this basic steps until all nodes have been connected. This algorithm is said to be "greedy" since it picks the immediate best option available without taking into account the long-term effects of the choices made. The algorithm is; start with any vertex, join this vertex to the nearest vertex, join on the vertex which is nearest to either of those already connected, repeat until all vertices are connected and add the lengths of all the edges included in the minimum length spanning tree.

The correctness of the algorithm follows directly from the fact that each arc that is added to the tree is contained in some minimum spanning tree with the arcs that have been selected in the previous step. The matrix formulation of Prim's algorithm implemented in this thesis is given below. 


\subsection{MATRIX FORMULATION OF PRIM'S ALGORITHM}

1. Choose a start vertex and delete all elements in that vertex's row and arrow its column.

2. Neglecting all deleted terms, scan all arrowed columns for the lowest element and circle that element.

3. Delete the circled element's row and arrow its column.

4. Repeat step 2 and 3 until all rows deleted.

5. The spanning tree is formed by the circled arc.

6. Write down the arcs in the order you selected them (Amponsah and Darkwah, 2007).

A company is installing a system of cables to connect all towns in the region. The lower case variables in the network show distances in kilometers. The question is what is the least amount of cable needed?

Figure 1: System of cables connecting cities

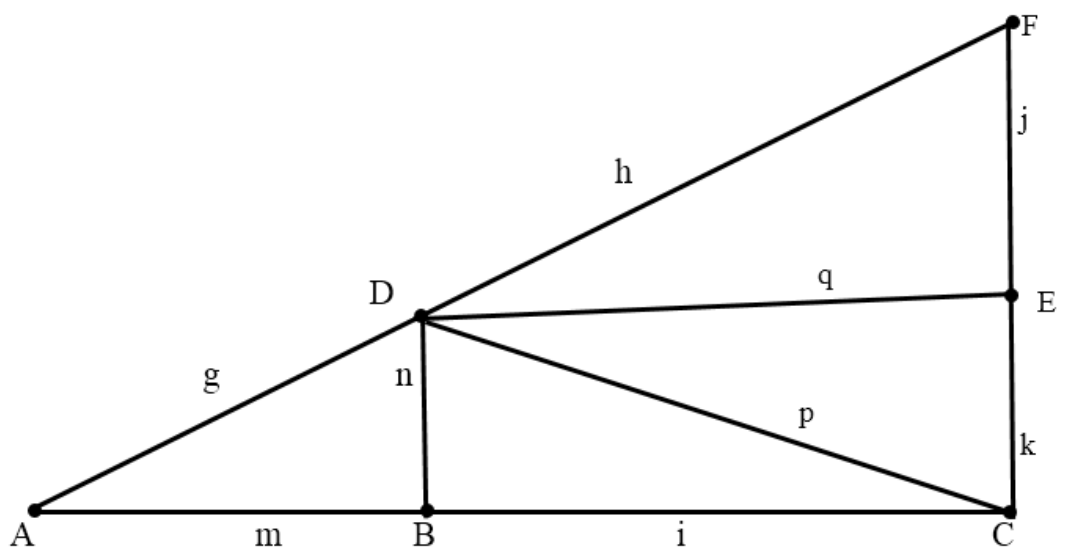

From Figure 1, it can be observed that Prim's algorithms work from a start point and builds up the spanning tree step by step, connecting edges into the existing solution. It can be applied directly to the distance matrix, as well as to work the network itself, so it is more suitable for using a computer if the network is large.

\subsection{RESULTS AND DISCUSSION}

The existing roads linking the regional capital towns are being used to provide access to the construction of the pipelines connection. The data involves length of road distances $(\mathrm{km})$ between the various regional capital towns. The methodology outlined in the chapter three will be used to identify the overall network layout from Takoradi to all the regional capital towns, the author's own optimal layout using Prim's algorithm and the environmental damages. For easy computation, we let A, B, C, D, E, F, G, H, I, and J represent Takoradi, Cape Coast, Accra, Ho, Koforidua, Kumasi, Sunyani, Wa, Tamale, and Bolgatanga respectively.

The pipelines used in these network systems can measure anywhere from 6 to 48 inches in diameter, although certain component pipe section can consist of small-diameter pipe that is as small as 0.5 inch in diameter. However, this small-diameter pipe is usually used only in gathering and distribution systems, although some is used for control-line or gauge-line purposes. Mainline pipes, the principal pipeline in a given system, are usually between 16 to 48 inches in diameter. Lateral pipelines, which deliver natural gas to or from the mainline, are typically between 6 and 16 inches in diameter. Most major interstate pipelines are between 24 and 36 inches in diameter (see Figure 2). 
Figure 2: Pipeline Network (in km) from Takoradi to all the regional capital towns in Ghana

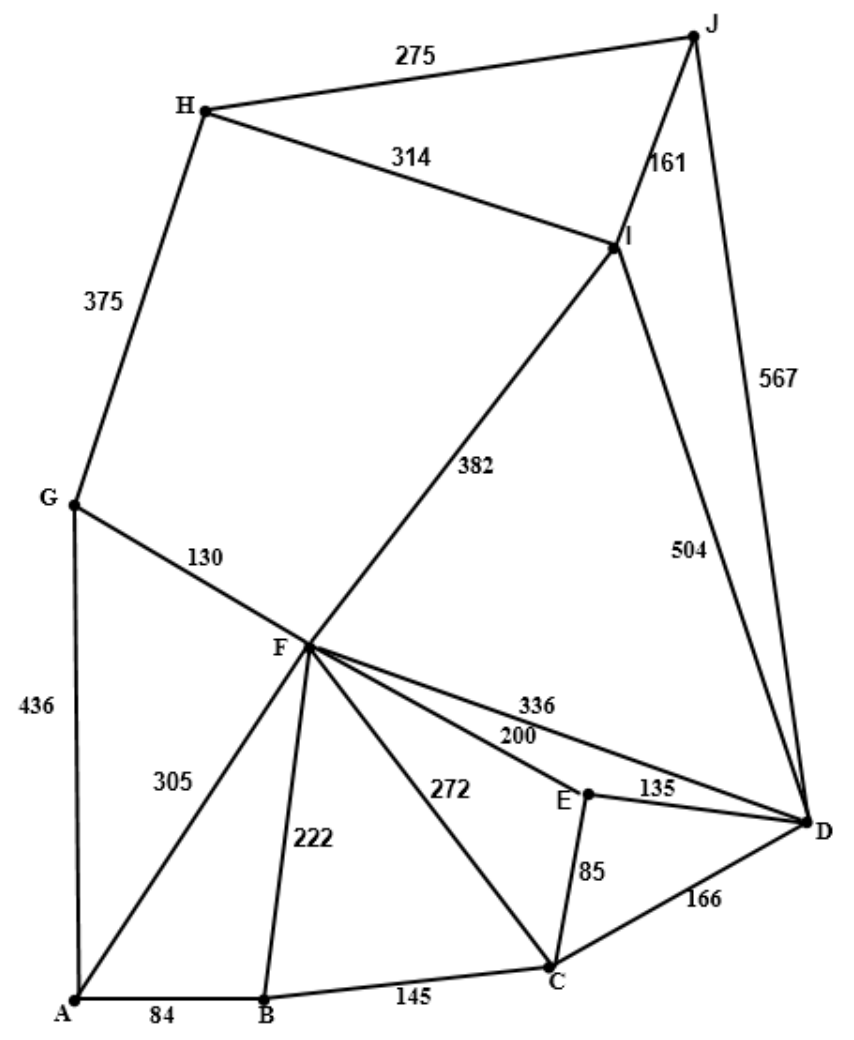

Total distance covered by the pipe network in the above map is 5,094km Source: Chana High Authority (Not drawn to scale)

Table 1: Distance matrix of the network

\begin{tabular}{|c|c|c|c|c|c|c|c|c|c|c|}
\hline & A & B & $\mathbf{C}$ & D & E & $\mathbf{F}$ & $\mathbf{G}$ & $\mathbf{H}$ & I & $\mathbf{J}$ \\
\hline A & $\infty$ & 84 & $\infty$ & $\infty$ & $\infty$ & 105 & 436 & $\infty$ & $\infty$ & $\infty$ \\
\hline B & 84 & $\infty$ & 145 & $\infty$ & $\infty$ & 222 & $\infty$ & $\infty$ & $\infty$ & $\infty$ \\
\hline C & $\infty$ & 145 & $\infty$ & 166 & 85 & 272 & $\infty$ & $\infty$ & $\infty$ & $\infty$ \\
\hline D & $\infty$ & $\infty$ & 166 & $\infty$ & 135 & 336 & $\infty$ & $\infty$ & 504 & 567 \\
\hline $\mathbf{E}$ & $\infty$ & $\infty$ & 85 & 135 & $\infty$ & 200 & $\infty$ & $\infty$ & $\infty$ & $\infty$ \\
\hline $\mathbf{F}$ & 305 & 222 & 272 & 336 & 200 & $\infty$ & 130 & $\infty$ & 382 & $\infty$ \\
\hline G & 436 & $\infty$ & $\infty$ & $\infty$ & $\infty$ & 130 & $\infty$ & 375 & $\infty$ & $\infty$ \\
\hline H & $\infty$ & $\infty$ & $\infty$ & $\infty$ & $\infty$ & $\infty$ & 375 & $\infty$ & 314 & 275 \\
\hline I & $\infty$ & $\infty$ & $\infty$ & 504 & $\infty$ & 382 & $\infty$ & 314 & $\infty$ & 161 \\
\hline $\mathbf{J}$ & $\infty$ & $\infty$ & $\infty$ & $\mathbf{5 6 7}$ & $\infty$ & $\infty$ & $\infty$ & 275 & 161 & $\infty$ \\
\hline
\end{tabular}

From Table 1, Prim's Algorithm is now applied to the distance matrix above with the solution taken from the graph. 


\subsection{MATRIX METHOD}

From Table 2, choose the starting vertex, A. Delete the row A. Look for the smallest entry in column A (see Figure 2).

Table 2: Stage 1 of the prims algorithm of the distance matrix

\begin{tabular}{|c|c|c|c|c|c|c|c|c|c|c|}
\hline & A & B & $\mathrm{C}$ & D & $\mathbf{E}$ & $\mathbf{F}$ & $\mathbf{G}$ & $\mathbf{H}$ & I & $\mathbf{J}$ \\
\hline $\mathrm{A}$ & & 84 & $\infty$ & $\infty$ & $\infty$ & 105 & -436 & $\infty$ & $-\infty$ & $\infty$ \\
\hline B & 84 & $\infty$ & 145 & $\infty$ & $\infty$ & 222 & $\infty$ & $\infty$ & $\infty$ & $\infty$ \\
\hline $\mathrm{C}$ & $\infty$ & 145 & $\infty$ & 166 & 85 & 272 & $\infty$ & $\infty$ & $\infty$ & $\infty$ \\
\hline D & $\infty$ & $\infty$ & 166 & $\infty$ & 135 & 336 & $\infty$ & $\infty$ & 504 & 567 \\
\hline $\mathbf{E}$ & $\infty$ & $\infty$ & 85 & 135 & $\infty$ & 200 & $\infty$ & $\infty$ & $\infty$ & $\infty$ \\
\hline $\mathbf{F}$ & 305 & 222 & 272 & 336 & 200 & $\infty$ & 130 & $\infty$ & 382 & $\infty$ \\
\hline G & 436 & $\infty$ & $\infty$ & $\infty$ & $\infty$ & 130 & $\infty$ & 375 & $\infty$ & $\infty$ \\
\hline $\mathbf{H}$ & $\infty$ & $\infty$ & $\infty$ & $\infty$ & $\infty$ & $\infty$ & 375 & $\infty$ & 314 & 275 \\
\hline I & $\infty$ & $\infty$ & $\infty$ & 504 & $\infty$ & 382 & $\infty$ & 314 & $\infty$ & 161 \\
\hline $\mathbf{J}$ & $\infty$ & $\infty$ & $\infty$ & 567 & $\infty$ & $\infty$ & $\infty$ & 275 & 161 & $\infty$ \\
\hline
\end{tabular}

From Table 3, it can be seen that $A B$ is the smallest edge joining $A$ to the other vertices. Put edge $A B$ into solution. Delete row B. Look for the smallest entry in columns A and B (see Figure 3). 
Table 3: Stage 2 of the Prims Algorithm of the Distance Matrix

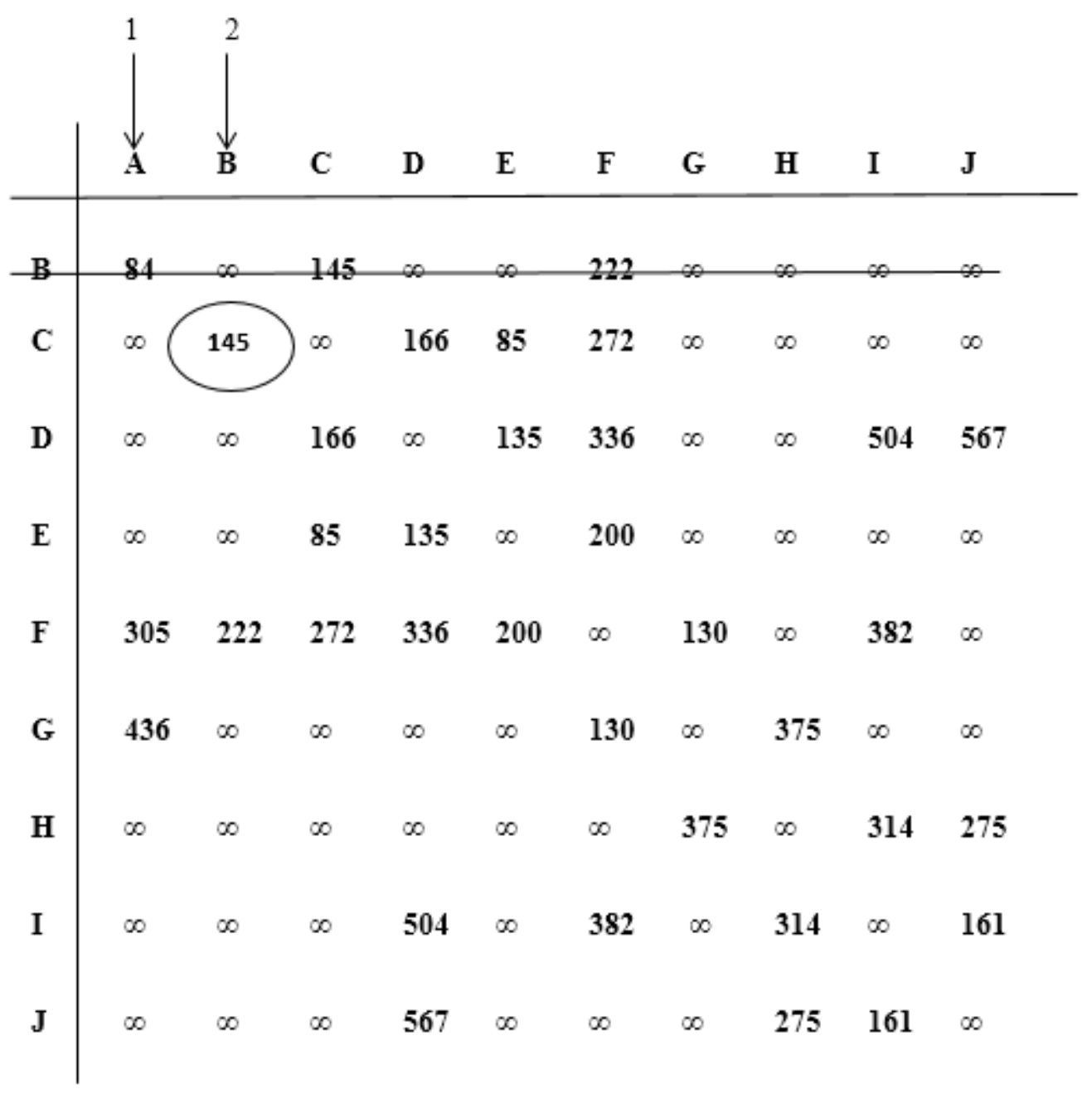

Figure 3: Solution 2

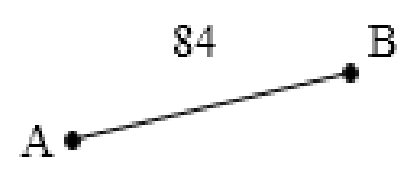

From Table 4, $B C$ is the smallest edge joining $A, B$ and $C$ to the other vertices. Put edge $B C$ into solution. Delete row $C$. Look for the smallest entry in columns A, B and C (see Figure 4). 
Table 4: Stage 3 of the prims algorithm of the distance matrix

\begin{tabular}{|c|c|c|c|c|c|c|c|c|c|c|}
\hline & $\begin{array}{l}1 \\
\downarrow \\
\mathbf{A}\end{array}$ & $\begin{array}{l}2 \\
\downarrow \\
\underset{B}{\downarrow}\end{array}$ & $\begin{array}{l}3 \\
\downarrow \\
\mathbf{C}\end{array}$ & D & $\mathbf{E}$ & $\mathbf{F}$ & G & $\mathbf{H}$ & I & $\mathbf{J}$ \\
\hline$-C$ & $\infty$ & 145 & $\infty$ & 166 & 85 & 272 & $\infty$ & $\infty$ & $\infty$ & $\infty$ \\
\hline D & $\infty$ & $\infty$ & 166 & $\infty$ & 135 & 336 & $\infty$ & $\infty$ & 504 & 567 \\
\hline $\mathbf{E}$ & $\infty$ & $\infty$ & 85 & 135 & $\infty$ & 200 & $\infty$ & $\infty$ & $\infty$ & $\infty$ \\
\hline $\mathbf{F}$ & 305 & 222 & 272 & 336 & 200 & $\infty$ & 130 & $\infty$ & 382 & $\infty$ \\
\hline G & 436 & $\infty$ & $\infty$ & $\infty$ & $\infty$ & 130 & $\infty$ & 375 & $\infty$ & $\infty$ \\
\hline $\mathbf{H}$ & $\infty$ & $\infty$ & $\infty$ & $\infty$ & $\infty$ & $\infty$ & 375 & $\infty$ & 314 & 275 \\
\hline I & $\infty$ & $\infty$ & $\infty$ & 504 & $\infty$ & 382 & $\infty$ & 314 & $\infty$ & 161 \\
\hline $\mathbf{J}$ & $\infty$ & $\infty$ & $\infty$ & 567 & $\infty$ & $\infty$ & $\infty$ & 275 & 161 & $\infty$ \\
\hline
\end{tabular}

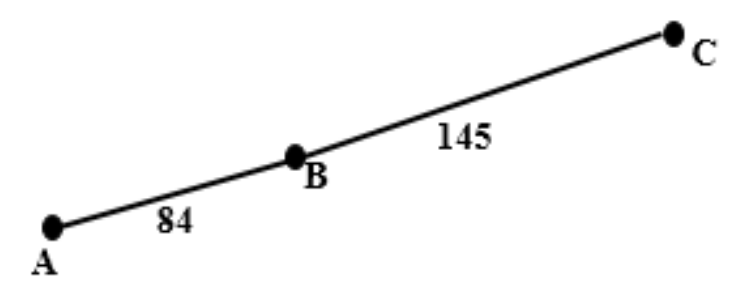

Table 5 presents $C E$ is the smallest edge joining $A, B, C$ and $E$ to the other vertices. Put edge $C E$ into solution. Delete row E. Look for the smallest entry in columns A, B, C and E (see Figure 5). 
Table 5: Stage 4 of the prims algorithm of the distance matrix

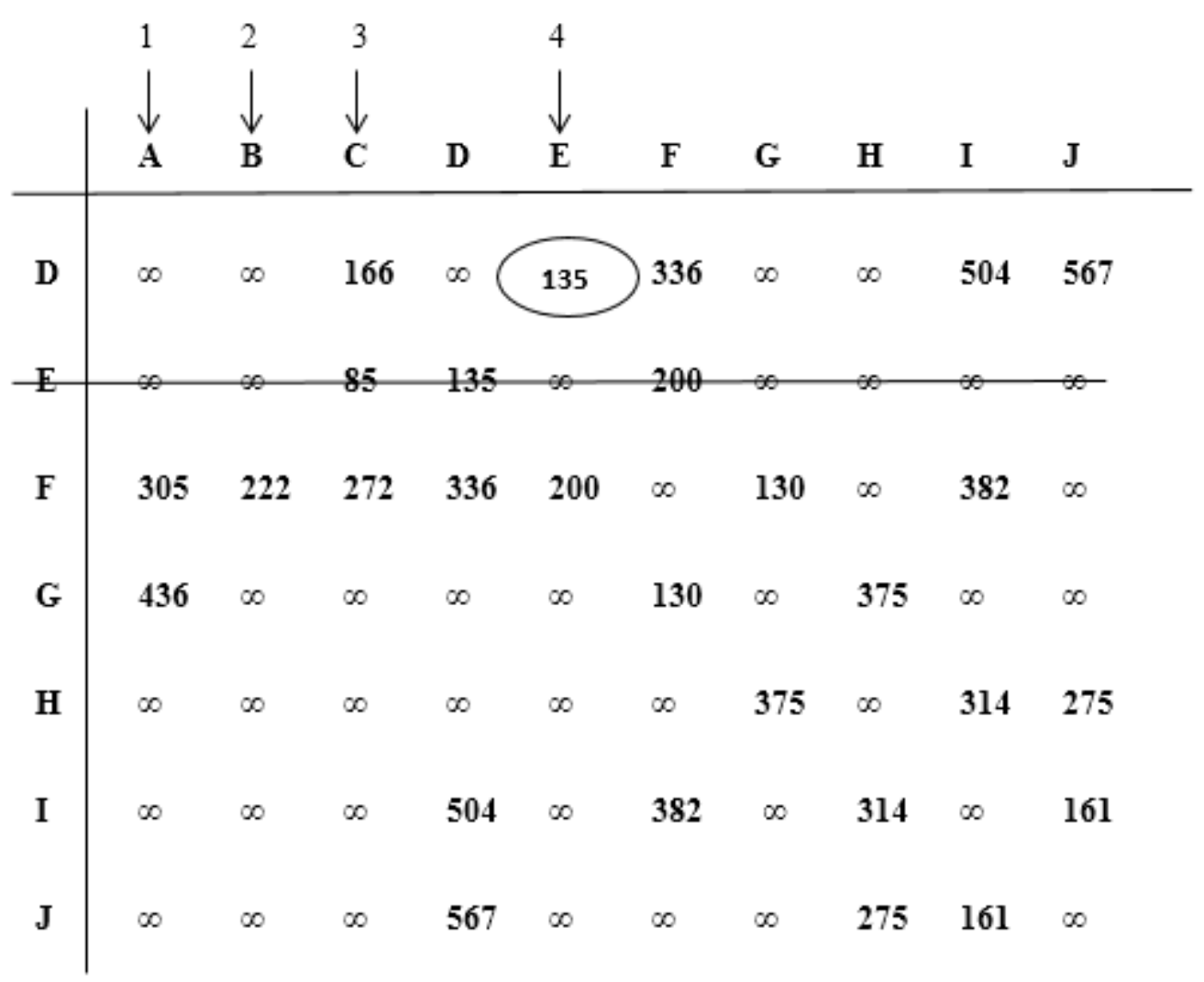

Figure 5: Solution 4

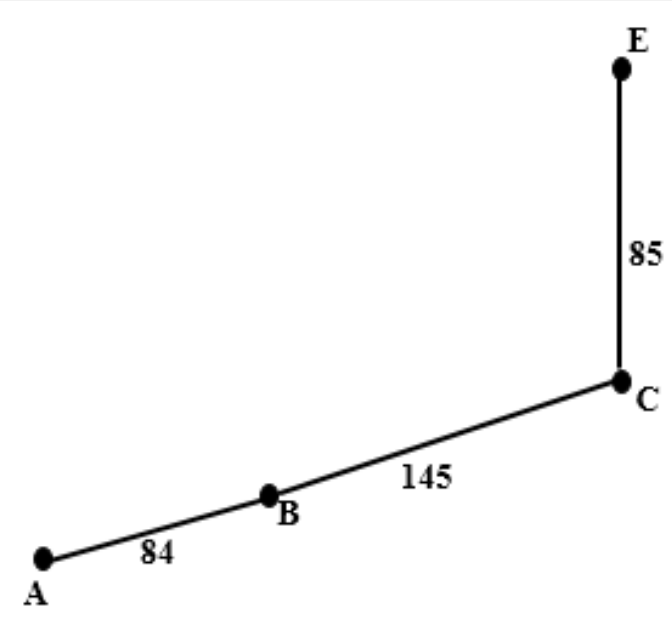

Table 6 and Figure 6 shows that ED is the smallest edge joining A, B, C, E and D to the other vertices. Put edge ED into solution. Delete row D. Look for the smallest entry in columns A, B, C, E and D as shown graphically in the below Figure 6 . 
Table 6: Stage 5 of the prims algorithm of the distance matrix
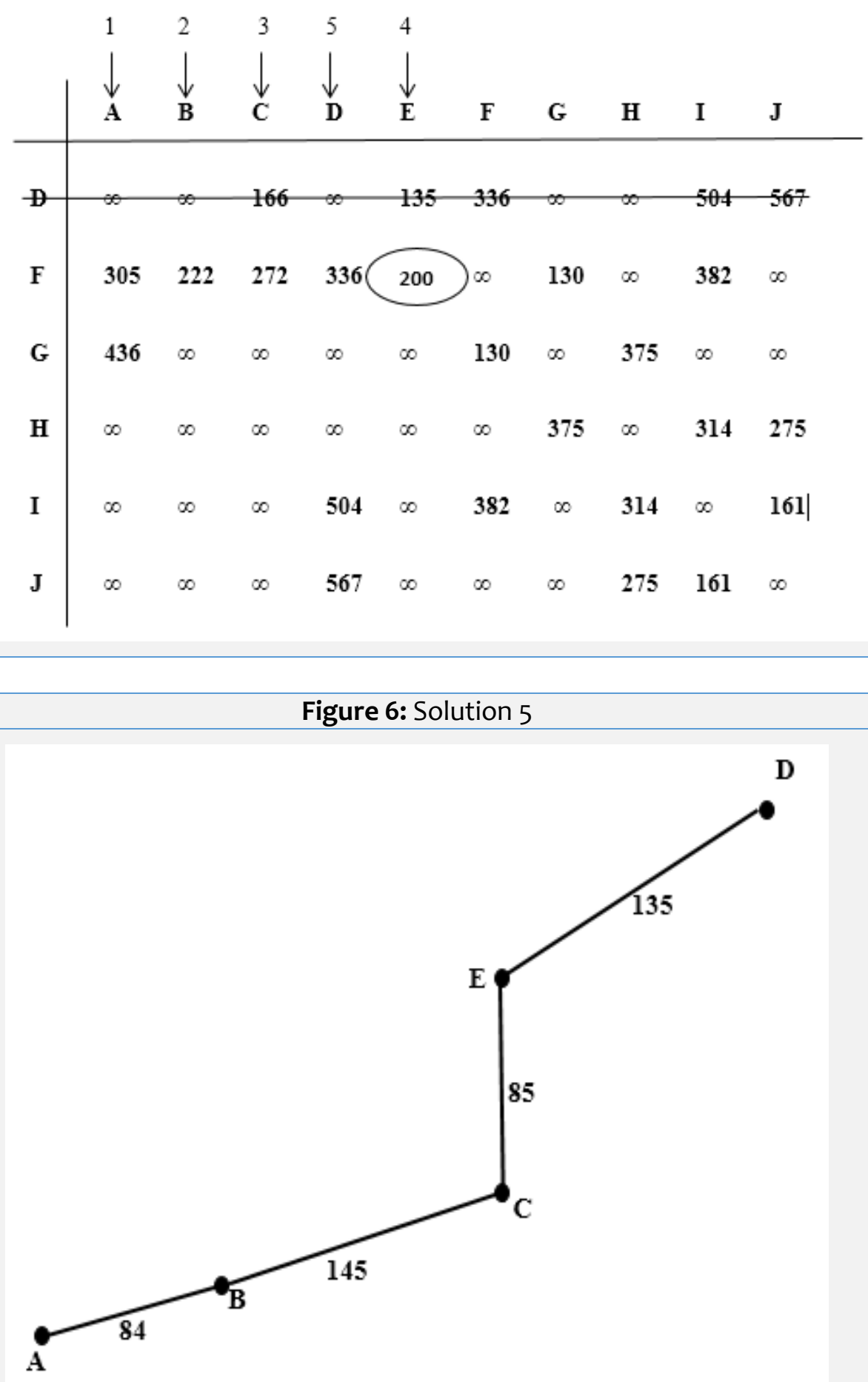

$D F$ is the smallest edge joining $A, B, C, E, D$ and $F$ to the other vertices. Put edge DF into solution. Delete row $\mathrm{F}$. Look for the smallest entry in columns $A, B, C, E, D$ and $F$ as shown in the below Table 7 and Figure 7 respectively. 
Table 7: Stage 6 of the prims algorithm of the distance matrix

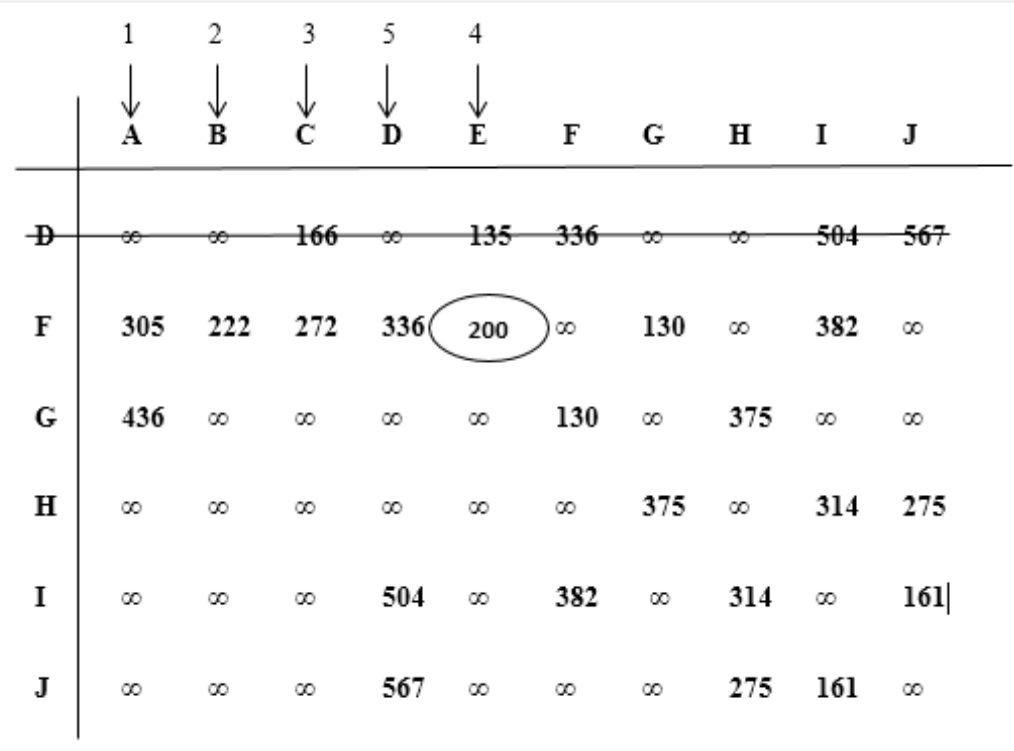

Figure 7: Solution 6

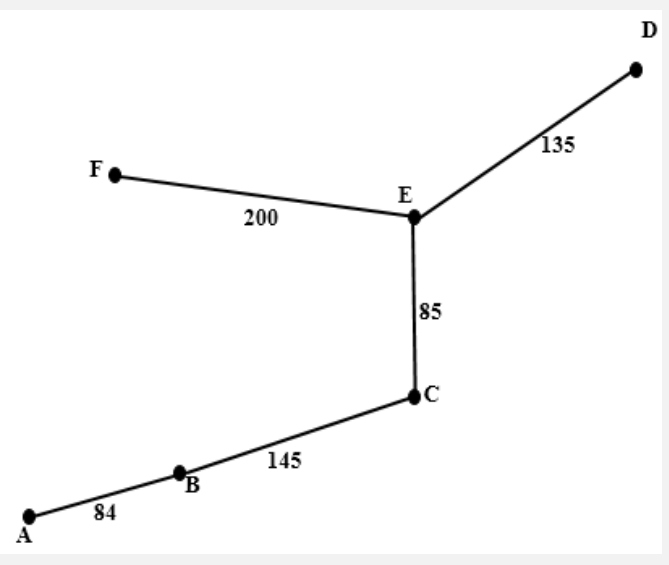

From Table 8, FG is the smallest edge joining A, B, C, E, D, F and G to the other vertices. Put edge FG into solution. Delete row $G$. Look for the smallest entry in columns A, B, C, E, D, F and G. This is shown in the below Figure 8.

Table 8: Stage 7 of the prims algorithm of the distance matrix

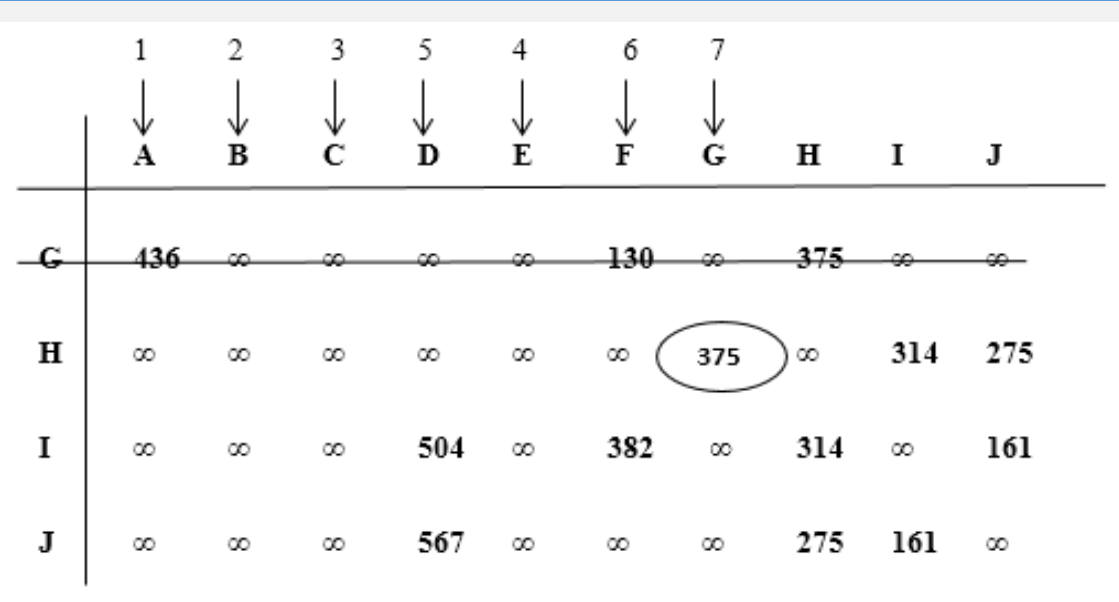


Figure 8: Solution 7

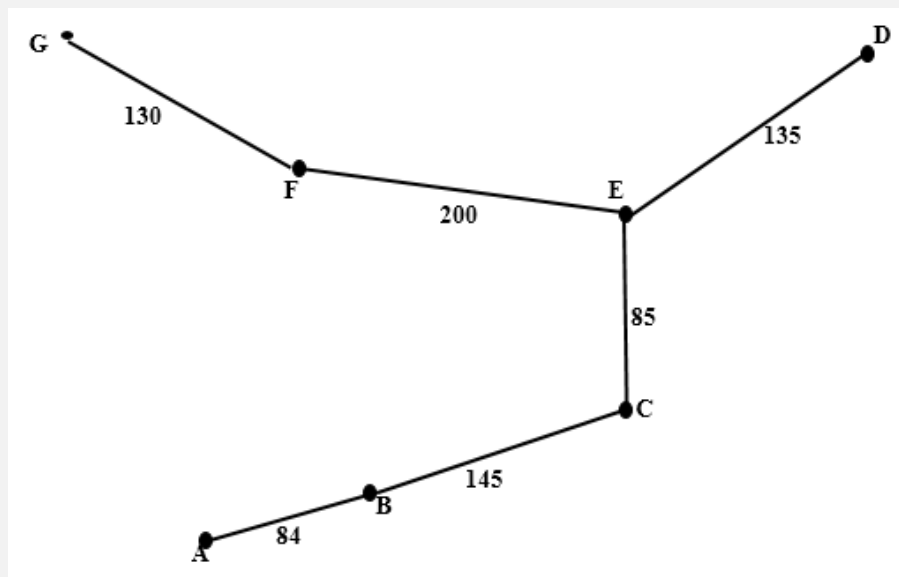

$\mathrm{GH}$ is the smallest edge joining $\mathrm{A}, \mathrm{B}, \mathrm{C}, \mathrm{E}, \mathrm{D}, \mathrm{F}, \mathrm{G}$ and $\mathrm{H}$ to the other vertices (see Table 9). Put edge $\mathrm{GH}$ into solution. Delete row H. Look for the smallest entry in columns A, B, C, E, D, F, G and H (see Figure 9).

Table 9: Stage 8 of the prims algorithm of the distance matrix

\begin{tabular}{|c|c|c|c|c|c|c|c|c|c|c|}
\hline & $\begin{array}{l}1 \\
\downarrow \\
\mathbf{A}\end{array}$ & $\begin{array}{l}2 \\
\downarrow \\
\text { b }\end{array}$ & $\begin{array}{l}3 \\
\downarrow \\
\mathbf{C}\end{array}$ & $\begin{array}{l}5 \\
\downarrow \\
\downarrow \\
\mathbf{D}\end{array}$ & $\begin{array}{l}4 \\
\downarrow \\
\mathbf{E}\end{array}$ & $\begin{array}{l}6 \\
\underset{\mathbf{F}}{\downarrow}\end{array}$ & $\begin{array}{l}7 \\
\underset{\mathbf{G}}{\downarrow}\end{array}$ & $\begin{array}{l}8 \\
\underset{\mathbf{H}}{\downarrow}\end{array}$ & I & $\mathbf{J}$ \\
\hline H & $\infty$ & $-\infty$ & $-\infty$ & $-\infty$ & $\infty$ & $\infty$ & 375 & $-\infty$ & 314 & 275 \\
\hline I & $\infty$ & $\infty$ & $\infty$ & 504 & $\infty$ & 382 & $\infty$ & 314 & $\infty$ & 161 \\
\hline $\mathbf{J}$ & $\infty$ & $\infty$ & $\infty$ & 567 & $\infty$ & $\infty$ & $\infty$ & 275 & 161 & $\infty$ \\
\hline
\end{tabular}

Figure 9: Solution 8

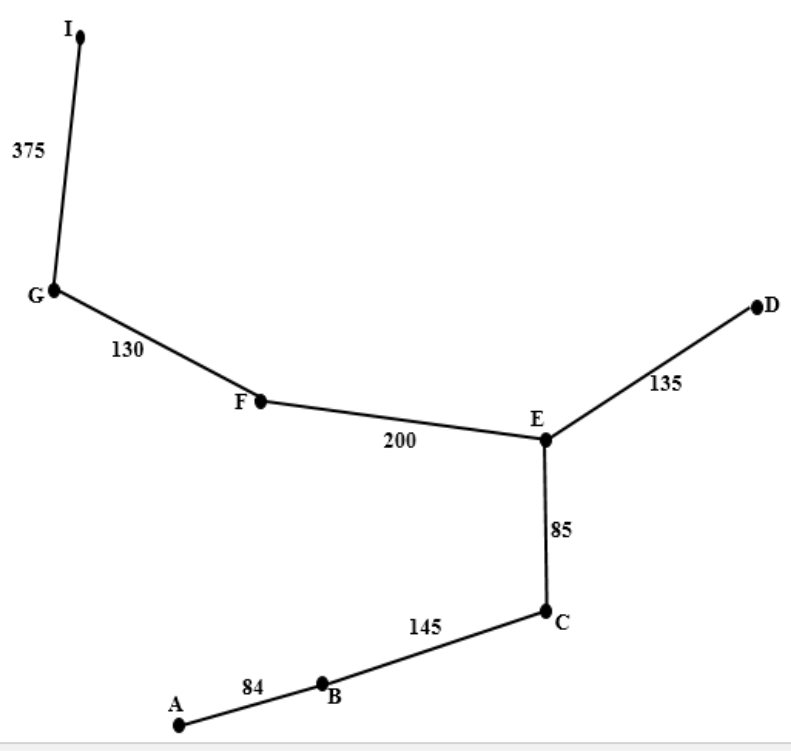


From Table 10, $\mathrm{HJ}$ is the smallest edge joining $\mathrm{A}, \mathrm{B}, \mathrm{C}, \mathrm{E}, \mathrm{D}, \mathrm{F}, \mathrm{G}, \mathrm{H}$ and $\mathrm{J}$ to the other vertices. Put edge $\mathrm{HJ}$ into solution. Delete row J. Look for the smallest entry in columns A, B, C, E, D, F, G, H and J (see Figure 10).

Table 10: Stage 9 of the prims algorithm of the distance matrix

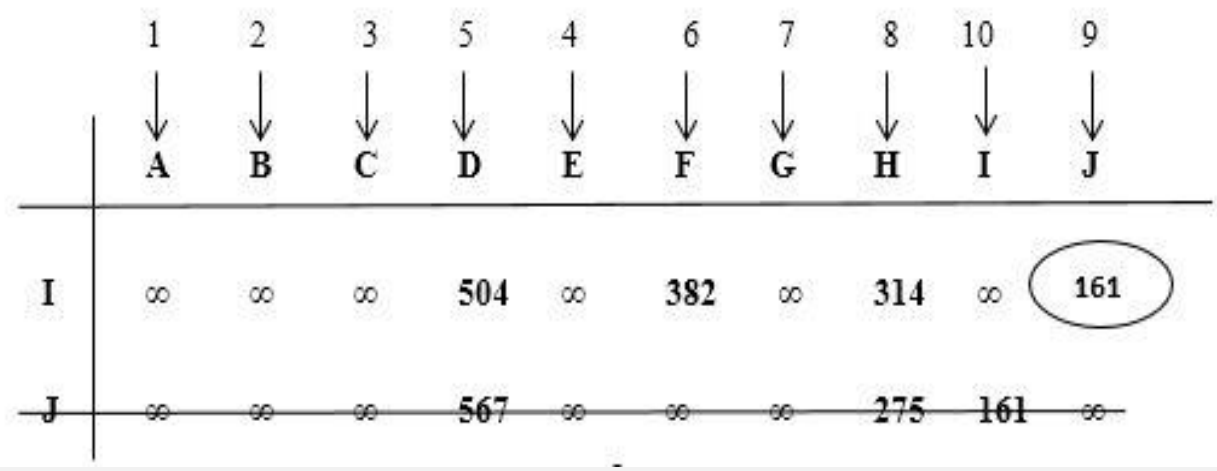

Figure 10: Solution 9

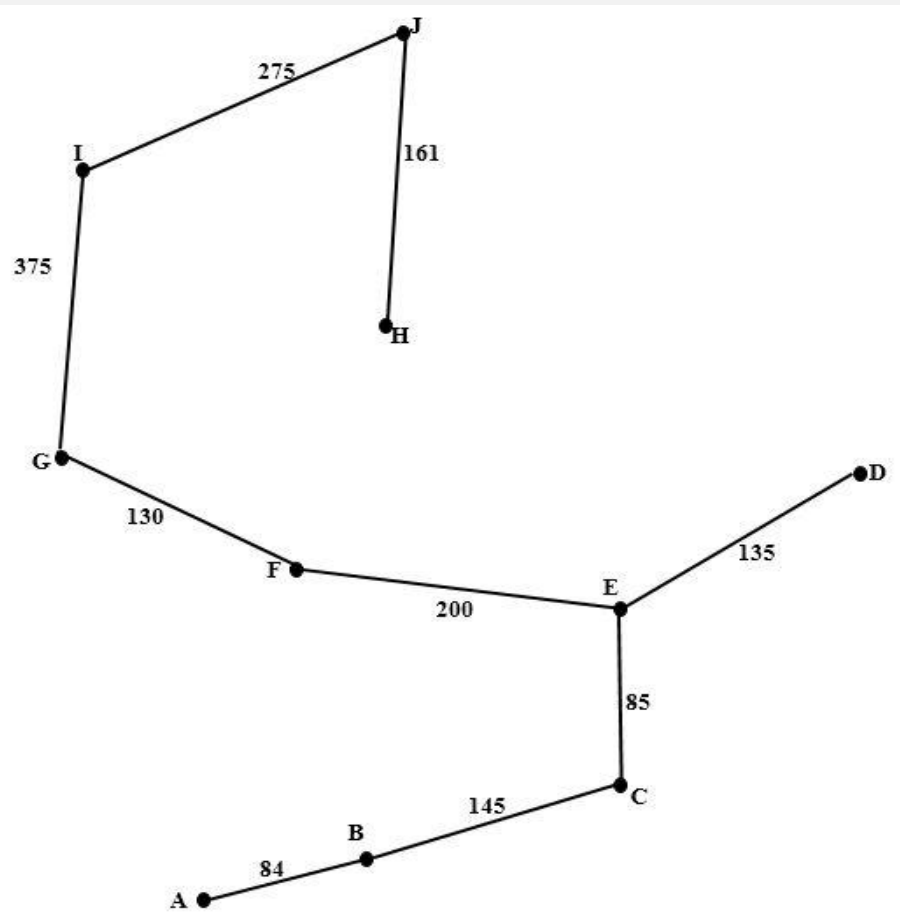

A look at Table 11 shows that $\mathrm{JI}$ is the smallest edge joining $\mathrm{A}, \mathrm{B}, \mathrm{C}, \mathrm{E}, \mathrm{D}, \mathrm{F}, \mathrm{G}, \mathrm{H}, \mathrm{J}$ and I to the other vertices. Put edge $\mathrm{Jl}$ into solution. This is shown pictorially in Figure 11.

Table 11: Final Stage of the Prims Algorithm of the Distance Matrix

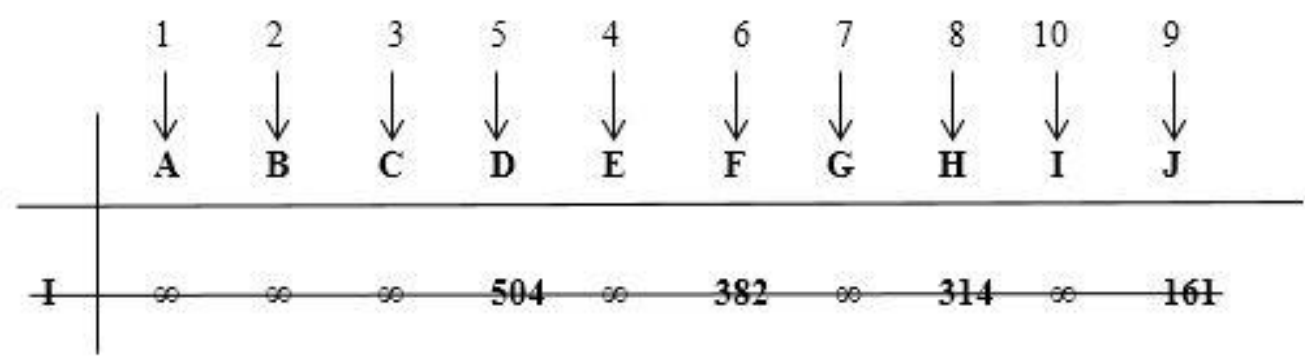


Figure 11: Solution 10

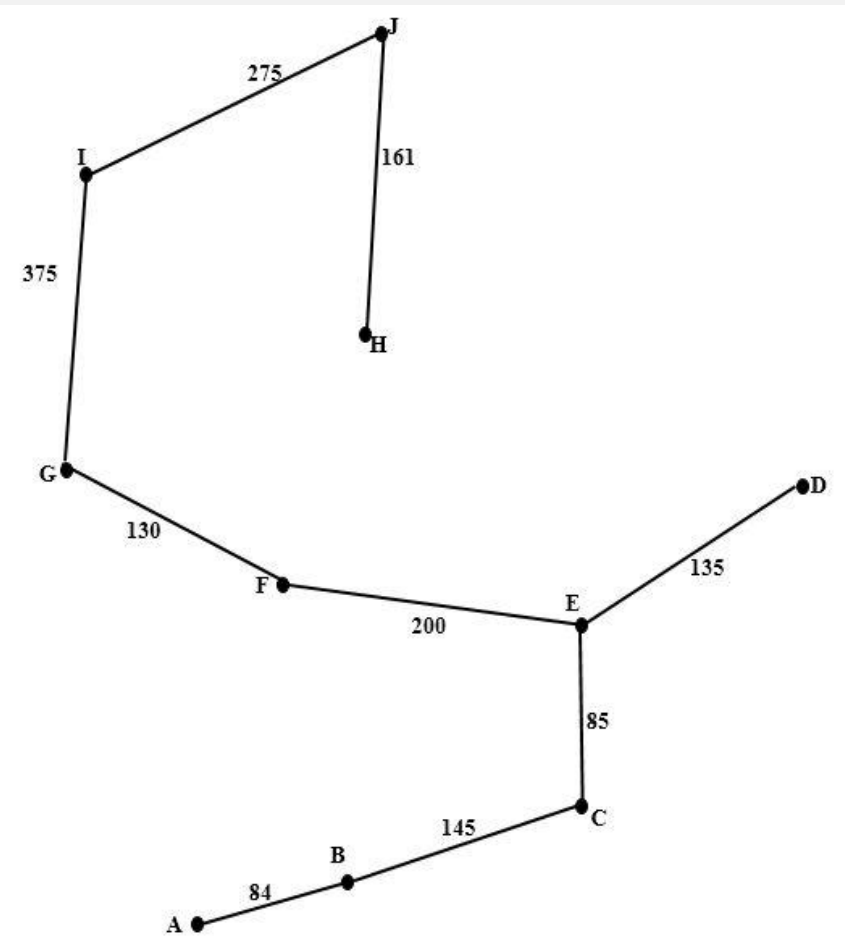

Table 12: Summary of Matrix Representation of the Pipeline Network.

\begin{tabular}{|c|c|c|c|c|c|c|c|c|c|c|}
\hline A & B & C & D & E & $\mathbf{F}$ & $\mathbf{G}$ & H & I & $\mathbf{J}$ & \\
\hline A & $\infty$ & 84 & $\infty$ & $\infty$ & $\infty$ & $\infty$ & $\infty$ & $\infty$ & $\infty$ & $\infty$ \\
\hline B & 84 & $\infty$ & 145 & $\infty$ & $\infty$ & $\infty$ & $\infty$ & $\infty$ & $\infty$ & $\infty$ \\
\hline C & $\infty$ & 145 & $\infty$ & $\infty$ & 85 & $\infty$ & $\infty$ & $\infty$ & $\infty$ & $\infty$ \\
\hline D & $\infty$ & $\infty$ & $\infty$ & $\infty$ & 135 & $\infty$ & $\infty$ & $\infty$ & $\infty$ & $\infty$ \\
\hline E & $\infty$ & $\infty$ & 85 & 135 & $\infty$ & 200 & $\infty$ & $\infty$ & $\infty$ & $\infty$ \\
\hline $\mathbf{F}$ & $\infty$ & $\infty$ & $\infty$ & $\infty$ & 200 & $\infty$ & 130 & $\infty$ & $\infty$ & $\infty$ \\
\hline G & $\infty$ & $\infty$ & $\infty$ & $\infty$ & $\infty$ & 130 & $\infty$ & 375 & $\infty$ & $\infty$ \\
\hline H & $\infty$ & $\infty$ & $\infty$ & $\infty$ & $\infty$ & $\infty$ & 375 & $\infty$ & $\infty$ & 275 \\
\hline I & $\infty$ & $\infty$ & $\infty$ & $\infty$ & $\infty$ & $\infty$ & $\infty$ & $\infty$ & $\infty$ & 161 \\
\hline $\mathbf{J}$ & $\infty$ & $\infty$ & $\infty$ & $\infty$ & $\infty$ & $\infty$ & $\infty$ & 275 & 161 & $\infty$ \\
\hline
\end{tabular}




\subsection{CONCLUSION}

The study provides an optimal solution for a trans-regional pipe line that provides economy. Before Prims Algorithm, the total distance covered by the pipe line network if the existing road networks are used from Takoradi to all the regional capitals towns in Ghana was 5,094km. By applying Prims Algorithm, the total distance covered is $1,590 \mathrm{~km}$. Considering the original map, total distance covered by the pipe network system has been reduced by $68.79 \%$.

It makes sense to argue that all other negative externalities in the form of environmental degradation which might relate to the construction of trans-regional gas pipeline might also be minimized by approximately $69 \%$.

\section{REFERENCES}

Amponsah, S. K., and Darkwah, F. K. (2007). Operation Research. KNUST press, Kumasi, Ghana. pp 2326.

Arogundade, O. T., and Akinwale A. T (2009). Application of Prim's Algorithm to a Profit- Oriented Transportation System in Rural Areas of Nigeria. University of Agriculture, Abeokuta, 37, 4-9.

Donkoh, E. K., Amponsah, S. K., Darkwa, K. F. (2011). Optimal pipeline connection for the West African Gas Pipeline Project. Research Journal of Applied Sciences, Engineering and Technology, 3 (2), 6773.

Prim, R. C., (1957). Shortest connection networks and some generalizations. Bell System Tech. Journal, 36, pp.1389-1401.

Hwee, M. Lin, R., Poe, W. A, (2007). Primary Reformer Operation: A Unique Application of Multivariable Control Nitrogen 230, pp35-41

WAGPCo, 2004a. Ghana Final Draft Rev 1. West African Gas Pipeline Environmental Impact Assessment. E981, 6: 47 .

WAGPCo, 2004b. Nigeria Final Draft Rev 1. West African Gas Pipeline Environmental Impact Assessment. E981, 7: 240-241.

WAGPCo, 2010. The Pipeline System. Retrieved http://www.wagpco.com/index.php?option=com_content\&view=article\&id=122\&ltemid $=85 \& l a l a=e n,($ Accessed on: November 10, 2010).

Wikipedia, 2008. Pipeline Transport. Retrieved from: http://en.wikipedia.org/wiki/Pipeline_transport, (Accessed on: November 31, 2010). 